\title{
Labyrinthe
}

21 | 2005 (2)

Communauté en pièces : d'Europe, d'Islam et d'ailleurs

\section{La horde d'or de 1377 à 1502}

Aux sources d'un siècle « sans Histoire »

\section{Marie Favereau}

\section{(2) OpenEdition}

\section{Journals}

Édition électronique

URL : http://journals.openedition.org/labyrinthe/918

DOI : $10.4000 /$ labyrinthe. 918

ISSN : 1950-6031

Éditeur

Hermann

Édition imprimée

Date de publication : 22 juin 2005

Pagination : 153-158

Référence électronique

Marie Favereau, «La horde d'or de 1377 à 1502 », Labyrinthe [En ligne], 21 | 2005 (2), mis en ligne le 16 juillet 2008, consulté le 22 septembre 2020. URL : http://journals.openedition.org/labyrinthe/918 ; DOI : https://doi.org/10.4000/labyrinthe.918

Propriété intellectuelle 


\title{
LA HORDE D'OR DE 1377 À 1502 Aux sources d'un siècle «sans Histoire »*
}

\author{
Marie FAVEREAU \\ mariefavereau@hotmail.com
}

Horde d'Or, Grande Horde, khanat de Qipčak, ulus de Jochi, Tartarie ou Mongolie d'Europe... Rarement dans l'histoire un État fut nommé de manière si variée. Ces multiples appellations sont révélatrices de la célébrité acquise par les descendants de Gengis Khan. Mais elles ne peuvent être dissociées d'un autre phénomène, qui caractérise le regard historiographique porté sur cet État depuis plus de deux siècles : la difficulté à appréhender une structure étatique complexe, que l'on pourrait qualifier de mouvante.

Ce que l'historiographie traditionnelle ${ }^{1}$ nomme Horde d'Or est un État qui s'étendait sur une grande partie de l'actuelle Russie, du Kazakhstan, de l'Ouzbékistan, du Turkménistan, du Caucase, de l'Ukraine et de la Bulgarie danubienne. Difficile, vu l'étendue de ces terres et leur fragmentation actuelle, de se représenter la Horde d'un point de vue géo-spatial. D'autant que cet État, constitué à partir des conquêtes occidentales de l'Empire mongol, et initialement formé par Jochi, fils aîné de Gengis Khan, dans les années vingt du XIII ${ }^{e}$ siècle, a connu par la suite quelques bouleversements géographiques: ainsi le Khorezm $^{2}$ et le Caucase, zones frontières que lui disputèrent les États mongols voisins (Ilkhanide et Chagatay), finirent par lui échapper - même si l'influence et l'autorité des souverains de la Horde continuèrent de peser sur l'ensemble de ces terres jusqu'à la fin du XVe siècle. De plus, une définition géographique de la Horde d'Or ne peut corres-

\footnotetext{
* Thèse de doctorat en Histoire médiévale soutenue le 10 décembre 2004 à l'université de Paris IV, sous la direction de M. Ludvik Kalus.

1. Parmi les ouvrages qui marquèrent leur temps: R. Grousset, L'Empire des steppes. Attila, Gengis Khan, Tamerlan, Paris, Payot, 1939; B. D. Grekiv, A. JU. Jakubovskij, Zolotaja Orda i ee padenie, Moscou-Saint Pétersbourg, 1950; G. V. Vernadskij, «The Mongols and Russia », A history of Russia, III, New Haven, Yale University Press, 1953; B. Spuler, Die Goldene Horde, die Mongolen in Rusland, 1223-1502, Wiesbaden, Otto Harrassowitz, 1943, rééd. 1965.

2. Région située au sud de la mer d'Aral.
} 
pondre à la définition territoriale que l'on donnerait d'un État contemporain. D'une part, la Horde comprenait de nombreuses cités, mais aucune capitale proprement dite; d'autre part, il est presque impossible de définir avec précision quelles furent ses limites géographiques (quelle frontière peut-on imposer au milieu d'un désert ou d'une steppe ?). Celles-ci doivent être déterminées en fonction de cités dont la mention sur une carte ancienne, une monnaie ou dans les textes, est souvent notre unique source pour mesurer l'intégration d'une région dans la Horde, ou la perte de cette même région, à une époque donnée.

La société de cet État n'est pas plus aisée à cerner. Dans les premiers temps, l'élite demeure influencée par son milieu d'origine, mongol et nomade, dont le système de représentations est structuré par le chamanisme ${ }^{3}$. Par la suite, les souverains de la Horde, les khans, vont se turciser et se convertir à l'Islam tout en conservant un certain nombre de règles issues de leurs ancêtres. L'organisation sociale de la Horde d'Or se caractérise donc par un syncrétisme complexe, qui mêle les apports successifs de plusieurs grands ensembles culturels. Les peuples qui vivaient sur les terres de cet État étaient nomades et sédentaires. On y parlait le persan, le türk (avec de multiples variantes dialectales), le slave, les langues du Caucase; on y pratiquait l'islam sunnite, la religion chrétienne selon plusieurs rites, le judaïsme et le chamanisme. Dans ces conditions, on comprend les difficultés rencontrées par tous les chercheurs qui se sont attelés à une reconstitution historique de la Horde d'Or. Malgré l'intérêt et même la fascination que les historiens vouent à cet État depuis le $\mathrm{XIX}^{\mathrm{e}}$ siècle, de nombreux aspects de son histoire demeurent dans l'obscurité et, aujourd'hui encore, définir avec clarté sa structure étatique et son organisation sociale relève de la gageure.

Le fil conducteur de nos recherches s'articule autour de la notion d'unité - redéfinie en dehors des canons d'appréciation propre à l'État nation - et s'inscrit contre l'incohérence de façade que semble renvoyer l'image de cette société. Cette incohérence apparente est en partie entretenue par la multiplicité des noms donnés à la Horde. Bien plus que le reflet des réalités sociales de l'époque, elle traduit l'essouffle-

3. B. Vladimircov, Obščestvennyj stroj Mongolov. Mongol'skij kočevojfeodalizm, Saint-Pétersbourg, 1934 ; Le Régime social des Mongols. Le féodalisme nomade, 1948; D. Aigle, «Le grand yasaq de Gengis-khan, l'empire, la culture mongole et la shari‘a », JESHO, 47/1, 2004, p. 51. 
ment et les désordres d'un discours historiographique qu'il est nécessaire de repenser. Redéfinir le rythme des articulations temporelles et établir l'existence d'une unité profonde de la société jochide sont les premières étapes de ce nouveau chantier historique ${ }^{4}$, fondé sur l'idée que la Horde d'Or est un modèle politique, social et économique dont la spécificité mérite d'être soulignée.

\section{Redéfinir le rythme des articulations temporelles}

Les éléments invariants du discours le plus traditionaliste sont fondés sur une périodisation de l'histoire de la Horde d'Or scandée en trois temps: 1) de la conquête au règne de Toqtamish (1223/24$1377)$; 2) du règne de Toqtamish à l'éclatement de la Horde (1377- v. 1430); 3) de l'émergence conflictuelle de khanats divisant la Horde à la destruction définitive de l'État (v. 1430-1502).

Les deux dernières périodes, celles du «déclin», sont considérées comme peu intéressantes, voire totalement passées sous silence. Ce délaissement généralisé s'explique, en particulier, par des raisons internes à l'historiographie russe dont l'un des thèmes de prédilection est, depuis le XIX ${ }^{e}$ siècle, l'asservissement au «joug tatar ${ }^{5}$ ». L'histoire de la Horde d'Or n'a, ainsi, jamais été réellement séparée de l'histoire étatique russe et continue aujourd'hui à jouer un rôle ambigu dans les représentations de l'identité nationale. De ce fait, à partir de la fin du XIVe siècle, on considère traditionnellement que la Horde entre dans la dernière phase de son histoire. Et le XV $\mathrm{XV}^{\mathrm{e}}$ siècle n'est perçu que sous le prisme de l'affaiblissement et du déclin de l'État khanial. Dans la continuité, il est établi que la Horde éclata en six khanats ${ }^{6}$ dans les années 1430: la Grande Horde, le khanat de Crimée, le khanat de Kazan, le khanat d'Astrakhan, le khanat de Kasymov et la Horde Shaybanide. Ce découpage géopolitique présente, à première vue, une sorte d'évidence historique trop confortable pour ne pas susciter nos interrogations. On voit que seraient déjà en formation, au $\mathrm{XV}^{\mathrm{e}}$ siècle, les grandes constitutions étatiques à venir:

4. La remise en cause du discours historiographique traditionnel et le renouvellement des débats concernant cette période de l'histoire ont été prônés par de nombreux chercheurs lors de la deuxième conférence internationale sur la Horde d'Or qui s'est tenue à Istanbul en avril 2005.

5. N. Karamzin, Histoire de l'empire de Russie, 11 tomes, 1819, Paris.

6. Ce terme, formé à partir du mot «khan » sur le modèle de sultan/sultanat, est utilisé dans les sources arabes et ne sera adopté que tardivement par les Turco-Mongols. 
le khanat de Crimée, les Shaybanides-Ouzbeks et le khanat de KazanTatarstan. Ces États ont de ce fait droit à une histoire, élément nécessaire d'un discours nationaliste. Les trois laissés pour compte: la Grande Horde, les khanats d'Astrakhan et de Kasymov, n'ayant pas connu de longévité historique et ne correspondant territorialement à aucun État contemporain, n'ont pas soulevé le même intérêt. Selon nous, l'hypothèse de l'existence d'un tel découpage étatique au début du $\mathrm{XV}^{\mathrm{e}}$ siècle repose sur trois principes: 1) les hordes ou khanats se définissent par de strictes assises territoriales; 2) les hordes ou khanats s'organisent autour d'un khan, ce qui suppose qu'il n'y a pas de khanat sans khan et que plusieurs khans ne peuvent être à la tête d'un même khanat; 3) les hordes ou khanats s'opposent sur les plans diplomatique et militaire: leurs politiques extérieures étant divergentes, aucun principe fédérateur ne permet de les considérer comme une entité.

Après une étude approfondie de l'ensemble des sources, on constate que ces trois principes doivent être remis en question. 1) L'autorité du khan est fondée non sur la possession légitime d'une terre, mais sur la soumission volontaire d'un ensemble de peuples. 2) Le pouvoir dans la Horde s'est constitué autour de figures fondatrices, principalement - mais non exclusivement - incarnées par des khans: on sait que la Horde fut dominée à plusieurs reprises par des chefs de confédérations tribales qui n'étaient pas khans; en outre, le pouvoir a toujours été partagé entre plusieurs descendants de Jochi, dont l'un avait préséance sur les autres. Le khan n'était pas considéré comme un souverain au pouvoir absolu mais comme un primus inter pares. 3) Les éléments unitaires et fédérateurs qui structuraient la Horde en tant qu'État sont perceptibles malgré l'apparent désordre des oppositions internes. L'équilibre étatique de la Horde ne doit, en effet, pas être appréhendé uniquement à travers les actions de l'élite politique qui la gouverne ${ }^{7}$. L'existence de référents identitaires et la force unificatrice des Institutions khaniales permettent de considérer la Horde comme une entité qui perdure malgré les dissensions personnelles de ses dirigeants.

La conception historique remise en cause ici est le fruit d'une démarche méthodologique fondée sur deux choix révélateurs de la position des historiens (qui ne se situent pas réellement comme historiens de la Horde d'Or) : d'une part, les dissensions internes à la Horde ont

7. D'autant plus que nous n'avons que très peu de sources directes sur ce point. 
trop souvent été appréhendées à travers un regard extérieur, influencé par une lecture partiale des sources et mené par d'autres enjeux que la compréhension du modèle politique et institutionnel jochide; d'autre part, on a eu clairement tendance à partir du fait politique et étatique qui caractérise les $\mathrm{XVI}^{\mathrm{e}}$-XVII ${ }^{\mathrm{e}}$ siècles pour expliquer et analyser les structures étatiques du $\mathrm{XV}^{\mathrm{e}}$ siècle. Le manque de sources et leur difficulté d'accès ont, bien entendu, contribué au développement de cette démarche «à contresens». Ainsi, l'émergence d'entités politiques nommées khanats et revendiqués comme tels par des souverains jochides doit être remise en question: l'existence de ces «khanats» correspond à une lecture faussée des mécanismes du pouvoir jochide et de ses assises. C'est en évitant de plaquer des problématiques propres à d'autres types de configuration étatique tels que le royaume, le sultanat ou la principauté, que nous pourrons mettre au jour les causes profondes de la force, puis de la décadence du modèle original de la Horde au $\mathrm{XV}^{\mathrm{e}}$ siècle.

\section{La force unificatrice des institutions}

En étudiant de manière approfondie les documents produits par la chancellerie des khans, nous pouvons réaliser à quel point la société jochide était structurée et cohérente. Ces documents se divisent en deux catégories: celle des yarliks, actes juridiques concernant l'administration intérieure de la Horde, et celle des bitiks, lettres diplomatiques adressées à des souverains étrangers. Leur étude nous a permis de définir le système juridique et administratif qui caractérise la Horde d'Or et la structure en tant qu'État. L'existence d'une production continue de yarliks et bitiks au $\mathrm{XV}^{\mathrm{e}}$ siècle montre qu'il y a incontestablement pérennité des institutions, et notamment de l'institution khaniale. Par ailleurs, la création de tels actes ne peut qu'être le fait d'un État organisé, possédant une administration extrêmement hiérarchisée, centralisée autour du souverain ${ }^{8}$. L'analyse de leurs textes, extrêmement construits, nous pousse à considérer le complexe administratif et social de la Horde comme un tout unique et cohérent. Par ailleurs, la complé-

8. D'autres productions étatiques viennent confirmer cette centralisation ou concentration autour de la figure khaniale: les monnaies, frappées au nom du khan, et les constructions monumentales (la 
mentarité de ces documents confirme un fait parfois mis en doute: la réalité d'une politique interne minutieuse et complexe menée sur plusieurs décennies par les khans. Enfin, notre étude nous a permis d'établir que trois systèmes judiciaires étaient en concurrence dans la Horde: l'un était administratif (yasaq), l'autre coutumier (yosun) et le dernier religieux ( $\left.\operatorname{sharî̀}^{\prime} a\right)$.

L'étude des pratiques juridiques khaniales qui découlent de la superposition des systèmes judiciaires nous confronte, une fois de plus, aux réalités complexes de cette société. Le syncrétisme qui la caractérise renvoie à l'historien l'image d'un monde éclaté et contradictoire. Il s'agit, pour donner sens à cette multiplicité, de repérer et d'isoler les divers éléments civilisationnels qui sont à l'origine de la Horde d'Or, pour ensuite dégager le lien, l'agencement très particulier qui structure sa société et lui confère une indéniable unicité.

L'organisation étatique de la Horde d'Or peut paraître extrêmement déroutante. Son étude révèle maintes lacunes liées à la méthodologie historique, et notamment un manque d'outils théoriques adaptés à une réflexion sur la notion d'État, en Asie centrale, à la période médiévale. L'analyse approfondie des structures sociales et de leurs représentations, perceptibles dans les sources, pourrait être l'une des voies à emprunter pour sortir de cette impasse. 